Notfall Rettungsmed 2018 $\cdot 21: 89$ https://doi.org/10.1007/s10049-018-0416-9 Online publiziert: 2. Februar 2018 (c) Springer Medizin Verlag $\mathrm{GmbH}$, ein Teil von Springer Nature 2018

CrossMark

Kindernotfälle stellen eine besondere Risiko- und Belastungssituation sowohl in der Präklinischen Versorgung als auch in der Notaufnahme dar. Die Ursache liegt darin, dass diese Notfälle einerseits relativ selten sind (ca. 5-10\% der Notarzteinsätze), andererseits aber durch Krankheitsbilder verursacht werden, die im Erwachsenenalter nicht vorkommen. Durch diese Konstellation ist ein Einüben des richtigen diagnostischen Vorgehens und der effizienten, leitliniengerechten Behandlung dieser Notfälle während der praktischen Tätigkeit im Notdienst kaum möglich.

I) Der Kindernotfall ist der am meisten gefürchtete Einsatzgrund

In verschiedenen Umfragen bei Notärzten und Rettungskräften wird immer wieder der Kindernotfall als der am meisten gefürchtete Einsatzgrund genannt. Infolgedessen werden im vorliegenden Heft mit dem Schwerpunkt Kindernotfälle mehrere Artikel vorgestellt, die eine Vorbereitung auf solche Notfallsituationen erlauben.

Bewusst wurden hier einerseits Abläufe dargestellt und ausgewählt, die bei Kindern anders abzuwickeln sind als bei den gewohnten Erwachsenennotfällen, andererseits aber auch ganz spezifisch nur Kinder betreffende Notfallsituationen, wie etwa die Geburt im Rettungsdienst und die Kindesmisshandlung. Alle Artikel sind so strukturiert, dass sie eine praktische Anwendung der dabei vorgestellten Konzepte ermöglichen.

Im Artikel „Interdisziplinäre Schockraumversorgung im Kindesalter" von Lehner et al. werden Algorithmen für die Versorgung von Kindern vorgestellt,

\title{
T. Nicolai
}

von Haunersches Kinderspital, Kinderklinik und Kinderpoliklinik, Ludwig Maximilian Universität München, München, Deutschland

\section{Kinder in Notfallsituationen}

deren Befolgung zu einer besseren Versorgungsqualität führt und ein besseres Training der versorgenden Teams erlaubt. Diese Algorithmen haben sich in der klinischen Praxis der beteiligten Autoren als sehr erfolgreich erwiesen und können auch als Handlungsschemata in Behandlungseinheiten platziert werden. $\mathrm{Zu}$ beachten sind die im Kindesalter anderen Verletzungsmuster sowie eine unter Nutzung des Ultraschalls im Vergleich zum Erwachsenen weniger stark auf frühes CT zentrierte Diagnostik.

Valter stellt zum Thema „Geburt im Rettungsdienst" typische Szenarien dieser für viele Erstversorger stressbehafteten Situation dar. Durch die derzeit steigenden Geburtenzahlen sind solche Ereignisse häufiger geworden. Eine normale Spontangeburt sowie typische Komplikationen, die sich präklinisch präsentieren können, und ihre Beherrschung sind hier dargestellt.

Ebenfalls im Zusammenhang mit der steigenden Geburtenrate ist der Artikel von Münch zum Thema „Notfälle bei außerklinischen Geburten" einzuordnen: Man wird häufiger zu Neugeborenen gerufen, die nach einer Hausgeburt in eine medizinische Notlage geraten sind. Hier liegt der Schwerpunkt nicht in der Geburtshilfe, sondern in der Behandlung der beeinträchtigt zur Welt kommenden Kinder. Der Artikel befasst sich auch mit der weiteren Versorgung und dem Transport des dann stabilisierten Neugeborenen und stellt typische spezielle Krankheitsbilder sowie ihre Behandlung vor.

Eine leider nicht zu vernachlässigende Zahl von Kindern wird durch Kindesmisshandlung und sog. nichtakzidentelle Traumata geschädigt. Bei Nichterkennung einer Kindesmisshandlung können sowohl der betroffene Patient als auch Ge- schwister von Wiederholungstaten und schwerster Schädigung bedroht sein.

In dem Artikel von Berthold et al. zum Thema „Kinderschutz im Rettungsdienst" werden Anleitungen zur Erkennung und zur korrekten Therapie vorgestellt. Auch wenn der präklinische Notarzt in der Regel keinen Beweis einer Kindesmisshandlung liefern kann, so kann er doch durch die Erkennung und Dokumentation entsprechender Verdachtsmomente rechtzeitig das weitere Vorgehen in die korrekte Richtung leiten.

Die folgenden sehr praktisch gehaltenen Übersichtsarbeiten erleichtern eine Vorbereitung auf Kindernotfälle und nehmen damit diesen an sich gut $\mathrm{zu}$ beherrschenden Situationen ein wenig das Angst- und Fehlerpotenzial.

\section{Muers}

\section{T. Nicolai}

\section{Korrespondenzadresse}

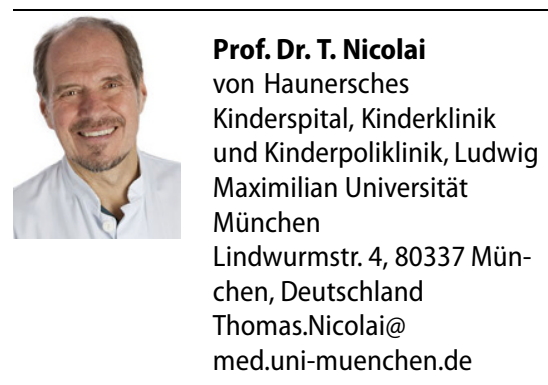

Interessenkonflikt. T. Nicolai gibt an, dass kein Interessenkonflikt besteht. 